\title{
CORRECT SELFADJOINT AND POSITIVE EXTENSIONS OF NONDENSELY DEFINED MINIMAL SYMMETRIC OPERATORS
}

\author{
I. PARASSIDIS AND P. TSEKREKOS
}

Received 13 May 2004

Let $A_{0}$ be a closed, minimal symmetric operator from a Hilbert space $\mathbb{U}$ into $\mathbb{U}$ with domain not dense in $\mathbb{U}$. Let $\hat{A}$ also be a correct selfadjoint extension of $A_{o}$. The purpose of this paper is (1) to characterize, with the help of $\hat{A}$, all the correct selfadjoint extensions $B$ of $A_{0}$ with domain equal to $D(\widehat{A}),(2)$ to give the solution of their corresponding problems, (3) to find sufficient conditions for $B$ to be positive (definite) when $\hat{A}$ is positive (definite).

\section{Introduction}

Minimal symmetric operators arise naturally in boundary value problems where they represent differential operators with all their defects, that is, their range is not the whole space and also their domain cannot be dense in the whole space. For example, the operator $A_{0}$ defined by the problem $A_{0} y=i y^{\prime}, y(0)=y(1)=0$ is a minimal symmetric nondensely defined operator. The problem of finding all correct selfadjoint extensions of a minimal symmetric operator is not either easy or always possible. The whole problem is facilitated when the domain of definition of the minimal symmetric operator is dense. Correct extensions of densely defined minimal not necessarily symmetric operators in Hilbert and Banach spaces have been investigated by Vishik [17], Dezin [3], Otelbaev et al. [10], Oı̆narov and Parasidi [14], and many others. Correct selfadjoint extensions of a densely defined minimal symmetric operator $A_{0}$ have been studied by a number of authors as J. Von Neumann [13], Kočubeř [7], Mikhaǔlets [12], and V. I. Gorbachuk and M. L. Gorbachuk [5]. They described the extensions as restrictions of some operators, usually of the adjoint operator $A_{0}^{*}$ of $A_{0}$. In this paper, we attack the above problem, developing a method which does not depend on maximal operators, but only on the existence of some correct selfadjoint extension of $A_{0}$. The essential ingredient in our approach is the extension of the main idea in [14]. More precisely, we show (Theorem 3.2) that every correct selfadjoint extension of a minimal operator is uniquely determined by a vector and a Hermitian matrix (see the comments preceding Theorem 3.2).

In $[1,2,9,8]$ extensions of nondensely defined symmetric operators by embedding $\mathbb{\square}$ in a space $X$ in which the operator $A_{0}$ is dense were studied. The class of extensions they 
consider is much wider than ours, but they do not consider correct selfadjoint extensions. Our method does not require such an embedding and applies equally well to positive correct selfadjoint extensions. Positive selfadjoint extensions of densely defined positive symmetric operators have been considered by Friedrichs [4].

As a demonstration of the theory developed in this paper, we give here all the correct selfadjoint extensions of the minimal operator $A_{0}$ in the example mentioned in the beginning of the introduction. These are the operators $B: L_{2}(0,1) \rightarrow L_{2}(0,1), B u=$ $i u^{\prime}-c t \int_{0}^{1} t u(t) d t$ with $D(B)=\{u \in \mathbb{H}(0,1): u(0)=-u(1)\}$, where $c$ is any real number.

The paper is organized as follows. In Section 2, we recall some basic terminology and notation about operators. In Section 3, we prove the main general results. Finally, in Section 4, we discuss several examples of integrodifferential equations which show the usefulness of our results.

\section{Terminology and notation}

By $\mathbb{U}$, we will always denote a complex Hilbert space with inner product $(\cdot, \cdot)$. The operators (linear) from $\mathbb{W}$ into $\mathbb{W}$ we refer to are not everywhere defined on $\mathbb{U}$. We write $D(A)$ and $R(A)$ for the domain and the range of the operator $A$, respectively. Two operators $A_{1}$ and $A_{2}$ are said to be equal if $D\left(A_{1}\right)=D\left(A_{2}\right)$ and $A_{1} x=A_{2} x$, for all $x \in D\left(A_{1}\right)$. $A_{2}$ is said to be an extension of $A_{1}$, or $A_{1}$ is a restriction of $A_{2}$, in symbol $A_{1} \subset A_{2}$ if $D\left(A_{2}\right) \supseteq D\left(A_{1}\right)$ and $A_{1} x=A_{2} x$, for all $x \in D\left(A_{1}\right)$. We notice that if $A \subset B$ and $A^{-1}, B^{-1}$ exist, then $A^{-1} \subset B^{-1}$. An operator $A_{0}: \mathbb{U} \rightarrow \mathbb{U}$ is called closed if for every sequence $x_{n}$ in $D(A)$ converging to $x_{0}$ with $A x_{n} \rightarrow f_{0}$, it follows that $x_{0} \in D(A)$ and $A x_{0}=f_{0}$. A closed operator $A_{0}: \mathbb{W} \rightarrow \mathbb{U}$ is called minimal if $R\left(A_{0}\right) \neq \mathbb{U}$ and the inverse $A_{0}^{-1}$ exists on $R\left(A_{0}\right)$ and is continuous. $A$ is called maximal if $R(A)=\mathbb{H}$ and $\operatorname{ker} A \neq\{0\}$. An operator $\hat{A}$ is called correct if $R(\hat{A})=\mathbb{H}$ and the inverse $\hat{A}^{-1}$ exists and is continuous. An operator $\hat{A}$ is called a correct extension (resp., restriction) of the minimal (resp., maximal) operator $A_{0}$ (resp., $A$ ) if it is a correct operator and $A_{0} \subset \widehat{A}$ (resp., $\widehat{A} \subset A$ ).

Let $A$ be an operator with domain $D(A)$ dense in $\mathbb{T}$. The adjoint operator $A^{*}: \mathbb{U} \rightarrow \mathbb{U}$ of $A$ with domain $D\left(A^{*}\right)$ is defined by the equation $(A x, y)=\left(x, A^{*} y\right)$ for every $x \in$ $D(A)$ and every $y \in D\left(A^{*}\right)$. The domain $D\left(A^{*}\right)$ of $A^{*}$ consists of all $y \in \mathbb{U}$ for which the functional $x \mapsto(A x, y)$ is continuous on $D(A)$. An operator $A$ is called selfadjoint if $A=A^{*}$ and symmetric if $(A x, y)=(x, A y)$ for all $x, y \in D(A)$. We note that, in the case in which $\overline{D(A)}=\mathbb{U}, A$ is symmetric if $A \subset A^{*}$. A symmetric operator $A$ is said to be positive if $(A x, x) \geq 0$ for every $x \in D(A)$ and positive definite if there exists a positive real number $k$ such that $(A x, x) \geq k\|x\|^{2}$, for all $x \in D(A)$.

The defect $\operatorname{def} A_{0}$ of an operator $A_{0}$ is the dimension of the orthogonal complement $R\left(A_{0}\right)^{\perp}$ of its range $R\left(A_{0}\right)$.

Let $F=\left(F_{1}, \ldots, F_{m}\right)$ be a vector of $\mathbb{a}^{m}$ and $A F=\left(A F_{1}, \ldots, A F_{m}\right)$. We write $F^{t}$ and $\left(A x, F^{t}\right)$ for the column vectors $\operatorname{col}\left(F_{1}, \ldots, F_{m}\right)$ and $\operatorname{col}\left(\left(A x, F_{1}\right), \ldots,\left(A x, F_{m}\right)\right)$, respectively. We denote by $\left(A F^{t}, F\right)$ the $m \times m$ matrix whose $i, j$ th entry is the inner product $\left(A F_{i}, F_{j}\right)$ and by $M^{t}$ the transpose matrix of $M$. We denote by $I$ and $\mathbf{0}$ the identity and the zero matrix, respectively. 


\section{Correct selfadjoint extensions of minimal symmetric operators}

Throughout this paper, $A_{0}$ will denote a nondensely defined symmetric minimal operator and $\hat{A}$ a correct selfadjoint extension of $A_{0}$. Let $E_{\mathrm{cS}}\left(A_{0}, \widehat{A}\right)$ denote the set of all correct selfadjoint extensions of $A_{0}$ with domain $D(\hat{A})$ and let $E_{\mathrm{cs}}^{m}\left(A_{0}, \hat{A}\right)$ denote the subset of $E_{\mathrm{cs}}\left(A_{0}, \hat{A}\right)$ consisting of all $B \in E_{\mathrm{cs}}\left(A_{0}, \hat{A}\right)$ such that $\operatorname{dim} R(B-\hat{A})=m$.

We begin with the following key lemma.

Lemma 3.1. For every $B \in E_{\mathrm{cs}}^{m}\left(A_{0}, \hat{A}\right)$, there exists a vector $F=\left(F_{1}, \ldots, F_{m}\right)$, where $F_{1}, \ldots, F_{m}$ are linearly independent elements of $D(\hat{A}) \cap R\left(A_{0}\right)^{\perp}$ and a Hermitian invertible matrix $T=$ $\left\|t_{i j}\right\|_{i, j=1}^{m}$ such that

$$
B x=\widehat{A} x-(\widehat{A} F) \bar{T} \overline{W^{-1}}\left(x, \widehat{A} F^{t}\right), \quad \forall x \in D(\widehat{A}),
$$

where $W=I+\left(\hat{A} F^{t}, F\right) T$, with $\operatorname{det} W \neq 0$.

Proof. Let $B \in E_{\mathrm{cs}}^{m}\left(A_{0}, \widehat{A}\right)$. Then $\operatorname{dim} R(B-\widehat{A})=m$. The main result of [10] implies that there exists a linear continuous operator $K: \mathbb{U} \rightarrow D(\hat{A})$ with $D(K)=\mathbb{H}$, $\operatorname{ker} K \supseteq R\left(A_{0}\right)$, $\operatorname{Ker}\left(\hat{A}^{-1}+K\right)=\{0\}$ such that

$$
B^{-1}=\hat{A}^{-1}+K \quad \text { or } \quad K=B^{-1}-\hat{A}^{-1} .
$$

Hence $K=K^{*}$, since $B^{-1}$ and $A^{-1}$ are selfadjoint operators. Since $A_{0}$ is a minimal operator, it follows that $R\left(A_{0}\right)$ is a closed subspace of $\mathbb{M}$, and so

$$
\mathbb{M}=R\left(A_{0}\right) \oplus R\left(A_{o}\right)^{\perp} \text {. }
$$

We will show that $\operatorname{dim} R(K)=m$. Indeed, from (3.2), it follows that $K f=B^{-1} f-\widehat{A}^{-1} f$ for all $f \in \mathbb{H}$. Let $x=B^{-1} f$. Then,

$$
x=\widehat{A}^{-1} f+K f, \quad \widehat{A} x=f+\widehat{A} K f,
$$

from which it follows that $(\hat{A}-B) x=\widehat{A}(K f)$, for all $f \in \mathbb{H}$. Since $\operatorname{dim} R(\hat{A}-B)=m$ and the operator $\hat{A}$ is invertible, we have $\operatorname{dim} R(K)=m$. Therefore, the selfadjointness of $K$ gives the decomposition

$$
\mathbb{H}=\operatorname{ker} K \oplus R(K) .
$$

From decompositions (3.3), (3.5), and the inclusion $\operatorname{ker} K \supseteq R\left(A_{0}\right)$, we conclude that

$$
R(K) \subseteq R\left(A_{0}\right)^{\perp}
$$

Fix a basis $\left\{F_{1}, F_{2}, \ldots, F_{m}\right\}$ of $R(K)$. Then, for every $f$ in $\mathbb{U}$, there are $\alpha_{i}$ in $\mathbb{R}$ such that

$$
K f=\sum_{i=1}^{m} \alpha_{i} F_{i} .
$$


Let $\left\{\psi_{1}, \psi_{2}, \ldots, \psi_{m}\right\}$ be the biorthogonal family of elements of $\mathbb{T}$ corresponding to the above basis of $R(K)$, that is, $\left(\psi_{i}, F_{j}\right)=\delta_{i j}, i, j=1, \ldots, m$. From $(3.7)$, we have $\left(K f, \psi_{j}\right)=$ $\left(\sum_{i=1}^{m} \alpha_{i} F_{i}, \psi_{j}\right)=\sum_{i=1}^{m} \alpha_{i}\left(F_{i}, \psi_{j}\right)=\alpha_{j}, j=1,2, \ldots, m$. Hence,

$$
K f=\sum_{i=1}^{m}\left(K f, \psi_{i}\right) F_{i}=\sum_{i=1}^{m}\left(f, K \psi_{i}\right) F_{i}, \quad \forall f \in \mathbb{H} .
$$

In particular, for $f=\psi_{j}$, we have

$$
K \psi_{j}=\sum_{i=1}^{m}\left(\psi_{j}, K \psi_{i}\right) F_{i}, \quad \text { or equivalently, } K \psi_{i}=\sum_{l=1}^{m}\left(\psi_{i}, K \psi_{l}\right) F_{l} .
$$

Replacing the above expression for $K \psi_{j}$ in (3.8), we obtain

$$
K f=\sum_{i=1}^{m}\left(f, \sum_{l=1}^{m}\left(\psi_{i}, K \psi_{l}\right) F_{l}\right) F_{i}=\sum_{i=1}^{m} \sum_{l=1}^{m}\left(f, F_{l}\right)\left(K \psi_{l}, \psi_{i}\right) F_{i} .
$$

If $T$ denotes the matrix $\left\|\left(K \psi_{l}, \psi_{i}\right)\right\|_{l, i=1}^{m}$, then (3.10) takes the form

$$
K f=F \bar{T}\left(f, F^{t}\right)=F \bar{T}\left(\overline{F^{t}, f}\right) .
$$

Now, the reader can easily verify that each of the matrices $T$ and $\left(\hat{A} F^{t}, F\right)$ is a Hermitian matrix. We claim that $T$ is invertible. Let $\hat{K}=\left.K\right|_{R(K)}$ denote the restriction of $K$ to its range. From (3.5), it follows that $\operatorname{ker} K \cap R(K)=\{0\}$. Therefore, $\operatorname{ker} \hat{K}=\{0\}$. Substituting $f=F_{j}$ into (3.11), we obtain

$$
K F_{j}=F \bar{T} \overline{\left(F^{t}, F_{j}\right)} \quad \text { or } \quad K F=F \bar{T} \overline{\left(F^{t}, F\right)} .
$$

The determinant $\operatorname{det}\left(F^{t}, F\right)$ is nonzero, being the determinant of the Gramm matrix $\left(F^{t}, F\right)$ of $F$. Since the vectors of $R(K) F_{1}, F_{2}, \ldots, F_{m}$ are linearly independent and $\operatorname{ker} \hat{K}=$ $\{0\}$, it follows that $\operatorname{det} T \neq 0$, which proves our claim.

We now prove the formula (3.1) which describes the action of the operator $B$ on $x$. From (3.4) and (3.11), we have

$$
\widehat{A} x=f+\widehat{A} F \bar{T} \overline{\left(F^{t}, f\right)}
$$

Then, taking the inner product with $F^{t}$, we get

$$
\begin{aligned}
\left(\widehat{A} x, F^{t}\right) & =\left(\widehat{A} F \bar{T} \overline{\left(F^{t}, f\right)}, F^{t}\right)+\left(f, F^{t}\right) \\
& =\overline{\left(F^{t}, \hat{A} F\right) \bar{T}} \overline{\left(F^{t}, f\right)}+\left(f, F^{t}\right) \\
& =\left(f, F^{t}\right)+\overline{\left(F^{t}, \hat{A} F\right)} \bar{T}\left(f, F^{t}\right) \\
& =\left[I+\overline{\left(\hat{A} F^{t}, F\right)} \bar{T}\right]\left(f, F^{t}\right) .
\end{aligned}
$$


Let $W$ denote the matrix $I+\left(\widehat{A} F^{t}, F\right) T$. We will show that $\operatorname{det} W \neq 0$. For if $\operatorname{det} W=$ 0 , then det $W^{t}=0$. Hence, there exists a nonzero vector $\vec{a}=\operatorname{col}\left(a_{1}, \ldots, a_{m}\right)$ such that $W^{t} \vec{a}=\vec{o}$. We consider the linear combination $f_{0}=\sum_{i=1}^{m} a_{i} \hat{A} F_{i}$. Since the vectors $F_{1}, \ldots, F_{m}$ are linearly independent and ker $\widehat{A}=\{0\}$, their images $\widehat{A}\left(F_{i}\right)$ under $\widehat{A}$ are linearly independent as well. It follows that $f_{0} \neq 0$. Combining (3.4) and (3.11), we get $x=\widehat{A}^{-1} f+$ $F \bar{T}\left(f, F^{t}\right)$, where $x=B^{-1} f$. In particular, for $x=B^{-1} f_{0}$, we compute

$$
\begin{aligned}
x_{0} & =\hat{A}^{-1} f_{0}+F \bar{T}\left(f_{0}, F^{t}\right) \\
& =\hat{A}^{-1} \sum_{i=1}^{m} \alpha_{i} \widehat{A} F_{i}+F \bar{T}\left(\sum_{i=1}^{m} \alpha_{i} \widehat{A} F_{i}, F^{t}\right) \\
& =F \vec{a}+F \bar{T} \sum_{i=1}^{m} \alpha_{i} \overline{\left(F^{t}, \widehat{A} F_{i}\right)} \\
& =F \vec{a}+F \bar{T} \sum_{i=1}^{m} \alpha_{i} \overline{\left(\hat{A} F^{t}, F_{i}\right)} \\
& =F I \vec{a}+F \bar{T} \overline{\left(\hat{A} F^{t}, F\right)} \vec{a} \\
& =F\left[I+\overline{T\left(\hat{A} F^{t}, F\right)}\right] \vec{a} \\
& =F W^{t} \vec{a} .
\end{aligned}
$$

In the above chain of equalities, the last one follows from the definition of $W$ and the fact that the matrices $T$ and $\left(\hat{A} F^{t}, F\right)$ are Hermitian. But $W^{t} \vec{a}=\overrightarrow{0}$. This implies that the nonzero vector $f_{0}$ is contained in the kernel $\operatorname{ker} B^{-1}$ of $B^{-1}$, contradicting the correctness of $B$. So det $W \neq 0$. Now (3.14) gives $\left(f, F^{t}\right)=\bar{W}^{-1}\left(x, \hat{A} F^{t}\right)$, which with (3.13) implies formula (3.1).

We now prove our main theorem which describes the set $E_{\mathrm{cs}}^{m}\left(A_{0}, \hat{A}\right)$ of all correct selfadjoint extensions $B$ of an operator $A_{0}$ with $D(B)=D(\hat{A})$ and $\operatorname{dim} R(B-\hat{A})=m$, using one correct selfadjoint extension $\hat{A}$ of a minimal symmetric operator $A_{0}$ with $\operatorname{def} A_{0} \leq \infty$. Every operator $B$ is uniquely determined by a vector $F$ with components $F_{i} \in D(\hat{A}) \cap$ $R\left(A_{0}\right)^{\perp}, i=1, \ldots, m$, and a Hermitian $m \times m$ matrix $C$ with rank $C=n \leq m$, satisfying condition (3.16) which is the solvability condition for the problem $B x=f$ (whose solution is also given in the following result).

Theorem 3.2. Suppose that $A_{0}, \hat{A}$ are as in Lemma 3.1. Then the following hold.

(i) For every $B \in E_{\mathrm{cs}}^{m}\left(A_{0}, \hat{A}\right)$, there exists a vector $F=\left(F_{1} \cdots F_{m}\right)$, where $F_{1}, \ldots, F_{m}$ are linearly independent elements from $D(\hat{A}) \cap R\left(A_{0}\right)^{\perp}$ and a Hermitian $m \times m$ matrix $C$ with $\operatorname{det} C \neq 0$, such that

$$
\begin{gathered}
\operatorname{det}\left[I-\overline{\left(\widehat{A} F^{t}, F\right)} C\right] \neq 0, \\
B x=\widehat{A} x-(\widehat{A} F) C\left(\widehat{A} x, F^{t}\right)=f .
\end{gathered}
$$

(ii) Conversely, for every vector $F=\left(F_{1} \cdots F_{m}\right)$, where $F_{1}, \ldots, F_{m}$ defined as above, and Hermitian $m \times m$ matrix $C$, which has rank $C=n \leq m$ and satisfies (3.16), the operator $B$ 
defined by (3.17) belongs to $E_{\mathrm{cs}}^{n}\left(A_{0}, \widehat{A}\right)$. The unique solution of (3.17) is given by the formula

$$
x=B^{-1} f=\hat{A}^{-1} f+F C\left[I-\overline{\left(\widehat{A} F^{t}, F\right)} C\right]^{-1}\left(f, F^{t}\right) \quad \forall f \in \mathbb{H} .
$$

Proof. (i) Let $B \in E_{\mathrm{cs}}^{m}\left(A_{0}, \widehat{A}\right)$. Then by Lemma 3.1, there exists a Hermitian, invertible $m \times$ $m$ matrix $T=\left(t_{i j}\right)$, and vector $F=\left(F_{1}, \ldots, F_{m}\right)$, where $F_{1}, \ldots, F_{m}$ are linearly independent elements from $D(\widehat{A}) \cap D\left(A_{0}\right)^{\perp}$ such that $\operatorname{det} W \neq 0$ and (3.1) holds true. From (3.1), since $B=B^{*}$, for every $y \in D\left(B^{*}\right)=D(B)=D(\widehat{A})$, we have

$$
\begin{aligned}
(B x, y) & =\left(\hat{A} x-(\hat{A} F) \bar{T} \bar{W}^{-1}\left(x, \widehat{A} F^{t}\right), y\right)=(\hat{A} x, y)-(\hat{A} F, y) \bar{T} \bar{W}^{-1}\left(x, \widehat{A} F^{t}\right) \\
& =(x, \hat{A} y)-\left(x, \overline{(\widehat{A} F, y) \bar{T} \bar{W}^{-1}}\left(\widehat{A} F^{t}\right)\right)=\left(x, \widehat{A} y-(y, \widehat{A} F) T W^{-1}\left(\widehat{A} F^{t}\right)\right)=\left(x, B^{*} y\right) .
\end{aligned}
$$

Hence,

$$
B^{*} y=\widehat{A} y-(y, \widehat{A} F) T W^{-1}\left(\widehat{A} F^{t}\right)=\widehat{A} y-(\widehat{A} F)\left(T W^{-1}\right)^{t}\left(y, \widehat{A} F^{t}\right)
$$

We denote by $C$ the matrix $\bar{T} \bar{W}^{-1}$. Since $B=B^{*}$, relations (3.1), (3.20) imply that

$$
C=\bar{T} \bar{W}^{-1}=\left(T W^{-1}\right)^{t}=\bar{C}^{t}
$$

Hence the matrix $C$ is Hermitian and so (3.1) implies (3.17). The invertibility of $C$ is implied by the fact that $\bar{T}$ and $\bar{W}^{-1}$ are invertible matrices. To show (3.16), we first remember that the $m \times m$ matrix $\left(\widehat{A} F^{t}, F\right)=D=\left(d_{i j}\right)$ is Hermitian. From $\bar{C}=T W^{-1}$, we take $T=\bar{C} W=\bar{C}(I+D T)$ or $\bar{C}=(I-\bar{C} D) T$. Since $\bar{C}$ and $T$ are invertible, it follows that $\operatorname{det}(I-\bar{C} D) \neq 0$, and we finally have that $\operatorname{det}(I-\bar{D} C) \neq 0$, that is, (3.16) is fulfilled.

(ii) We will show that $B \in E_{\mathrm{cs}}^{n}\left(A_{0}, \hat{A}\right)$. We first show that $B$ is a correct extension of $A_{0}$. Taking into account (3.17), we have

$$
\begin{aligned}
\left(F^{t}, f\right) & =\left(F^{t}, \widehat{A} x-(\widehat{A} F) C\left(\widehat{A} x, F^{t}\right)\right) \\
& =\left[I-\left(\widehat{A} F^{t}, F\right) \bar{C}\right] \overline{\left(\widehat{A} x, F^{t}\right)}
\end{aligned}
$$

or

$$
\left[I-\overline{\left(\widehat{A} F^{t}, F\right)} C\right]\left(\widehat{A} x, F^{t}\right)=\left(f, F^{t}\right)
$$

From (3.16), we have

$$
\left(\widehat{A} x, F^{t}\right)=\left[I-\overline{\left(\widehat{A} F^{t}, F\right)} C\right]^{-1}\left(f, F^{t}\right) .
$$


Since $\hat{A}$ is invertible, (3.17) implies that

$$
x-F C\left(\hat{A} x, F^{t}\right)=\hat{A}^{-1} f, \quad f=B x,
$$

and because of (3.24), we have

$$
x=\widehat{A}^{-1} f+F C\left[I-\overline{\left(\hat{A} F^{t}, F\right)} C\right]^{-1}\left(f, F^{t}\right), \quad \forall f \in \mathbb{H},
$$

which is (3.18).

Since $\hat{A}^{-1}$ is continuous on $\mathbb{U}, B^{-1}$ is continuous on $\mathbb{W}$. From (3.18), it is clear that $D(B)=D(\hat{A}) \supseteq D\left(A_{0}\right)$. Since $A_{0} \subset \widehat{A}$ and $F_{i} \in R\left(A_{0}\right)^{\perp}, i=1, \ldots, m$, it follows from (3.17) that $B x=\widehat{A} x=A_{0} x$, for all $x \in D\left(A_{0}\right)$.

So, $A_{0} \subset B$ and since $B^{-1}$ exists and is continuous on $\mathbb{M}, B$ is a correct extension of $A_{0}$. From (3.17), because of rank $C=n$ and $\widehat{A} F_{1}, \ldots, \widehat{A} F_{m}$ being linearly independent, it follows that $\operatorname{dim} R(B-\hat{A})=n$.

It remains to show that $B=B^{*}$.

Taking into account (3.17) for $y \in D(\widehat{A})$, we have

$$
\begin{aligned}
(B x, y) & =(\widehat{A} x, y)-\left((\widehat{A} F) C\left(\widehat{A} x, F^{t}\right), y\right)=(x, \widehat{A} y)-(\widehat{A} F, y) C\left(\widehat{A} x, F^{t}\right) \\
& =(x, \widehat{A} y)-\left(x, \overline{(\widehat{A} F, y) C}\left(\widehat{A} F^{t}\right)\right)=\left(x, \widehat{A} y-(y, \widehat{A} F) \bar{C}\left(\widehat{A} F^{t}\right)\right)=(x, \phi) .
\end{aligned}
$$

It follows that $y \in D\left(B^{*}\right)$ and $D(\widehat{A})=D(B) \subseteq D\left(B^{*}\right)$. But for $y \in D(\widehat{A})$, we have

$$
B^{*} y=\phi=\widehat{A} y-(y, \widehat{A} F) \bar{C}\left(\widehat{A} F^{t}\right)=\widehat{A} y-(\widehat{A} F) C\left(\widehat{A} y, F^{t}\right)=B y .
$$

Hence $B \subset B^{*}$. Let now $y \in D\left(B^{*}\right)$. From (3.17), we have

$$
\begin{aligned}
& (B x, y)=(\widehat{A} x, y)-\left((\widehat{A} F) C\left(\hat{A} x, F^{t}\right), y\right)=(\widehat{A} x, y)-(\widehat{A} F, y) C\left(\widehat{A} x, F^{t}\right) \\
& =\left(\hat{A} x, y-\overline{(\hat{A} F, y) C} F^{t}\right)=\left(x, B^{*} y\right) .
\end{aligned}
$$

So, $y-\overline{(\hat{A} F, y) C} F^{t} \in D\left(\hat{A}^{*}\right)=D(\widehat{A})=D(B)$ and since $F_{1}, \ldots, F_{m} \in D(\hat{A})$, it follows that $y \in D(\hat{A})$. Hence, $D\left(B^{*}\right)=D(\hat{A})=D(B)$ and $B=B^{*}$. So the theorem has been proved.

In the next particular case when $F_{i} \in D\left(A_{0}\right) \cap R\left(A_{0}\right)^{\perp}, i=1, \ldots, m$, the condition (3.16) is fulfilled automatically and the solution of $B x=f$ is simpler. 
Corollary 3.3. For every vector $F=\left(F_{1} \cdots F_{m}\right)$, where $F_{1}, \ldots, F_{m}$ are linearly independent elements from $D\left(A_{0}\right) \cap R\left(A_{0}\right)^{\perp}$, and for every Hermitian $m \times m$ matrix $C$ with rank $C=$ $n \leq m$, the operator $B$ defined by (3.17) belongs to $E_{\mathrm{cs}}^{n}\left(A_{0}, \widehat{A}\right)$.

The unique solution of (3.17) is given by

$$
x=B^{-1} f=\widehat{A}^{-1} f+F C\left(f, F^{t}\right), \quad \forall f \in \mathbb{M} .
$$

Proof. Indeed, if $F_{i} \in D\left(A_{0}\right) \cap R\left(A_{0}\right)^{\perp}, i=1, \ldots, m$, then $\left(\widehat{A} F_{i}, F_{j}\right)=\left(A_{0} F_{i}, F_{j}\right)=0$ for all $i, j=1, \ldots, m$, since $F_{j} \in R\left(A_{0}\right)^{\perp}, j=1, \ldots, m$. Hence $\left(\hat{A} F^{t}, F\right)=\mathbf{0}$. The rest easily follows from the above theorem.

Remark 3.4. For every $B \in E_{\mathrm{cs}}^{m}\left(A_{0}, \hat{A}\right)$ from (3.2) and (3.6), we have

$$
R\left(B^{-1}-\hat{A}^{-1}\right) \subseteq R\left(A_{0}\right)^{\perp}, \quad \operatorname{dim} R(B-\widehat{A})=m \leq \operatorname{def} A_{0} .
$$

Let now the minimal operator $A_{0}$ have finite defect $\operatorname{def} A_{0}=\operatorname{dim} R\left(A_{0}\right)^{\perp}=m$. Then $D\left(A_{0}\right)$ can be defined as follows:

$$
D\left(A_{0}\right)=\left\{x \in D(\widehat{A}):\left(\widehat{A} x, F^{t}\right)=\mathbf{0}\right\}
$$

where $F=\left(F_{1} \cdots F_{m}\right), F_{1}, \ldots, F_{m}$ are linearly independent elements of $R\left(A_{0}\right)^{\perp} \cap D(\hat{A})$. So if we have chosen the elements $F_{1}, \ldots, F_{m}$ so that (3.32) holds, then every $B$ from $E_{\mathrm{cs}}^{m}\left(A_{0}, \hat{A}\right)$ is defined only by the Hermitian matrix $C$ and we can restate Theorem 3.2 as follows.

Theorem 3.5. (i) For every $B \in E_{\mathrm{cs}}^{m}\left(A_{0}, \hat{A}\right)$, where $A_{0}$ satisfies (3.32), there exists a Hermitian $m \times m$ matrix $C$ with $\operatorname{det} C \neq 0$, such that (3.16) and (3.17) are fulfilled.

(ii) Conversely, for every Hermitian $m \times m$ matrix $C$, which satisfies (3.16) and rank $C=n$, the operator $B$ defined by (3.17) belongs to $E_{\mathrm{cs}}^{n}\left(A_{0}, \hat{A}\right)$. The unique solution of (3.17) is given by (3.18).

Proof. From (3.32), we have

$$
R\left(A_{0}\right)=\left\{f \in \mathbb{U}:\left(f, F_{i}\right)=0, i=1, \ldots, m\right\}
$$

It is evident that $\operatorname{dim} R\left(A_{0}\right)^{\perp}=m$ and $\left\{F_{1}, \ldots, F_{m}\right\}$ is a basis of $R\left(A_{0}\right)^{\perp}$. Then from $\operatorname{dim} R\left(A_{0}\right)^{\perp}=m, \operatorname{dim} R(K)=m$, and (3.6), it follows that

$$
R(K)=R\left(B^{-1}-\hat{A}^{-1}\right)=R\left(A_{0}\right)^{\perp} .
$$

As basis of $R(K)$, we can take $F_{1}, \ldots, F_{m}$. The rest is proved similarly.

Remark 3.6. For every $B \in E_{\mathrm{cs}}^{m}\left(A_{0}, \widehat{A}\right)$, where $A_{0}$ satisfies (3.32), we have $R\left(B^{-1}-\widehat{A}^{-1}\right)=$ $R\left(A_{0}\right)^{\perp}$ and $\operatorname{dim} R(B-\widehat{A})=\operatorname{def} A_{0}$. 
Remark 3.7. The operators $B \in E_{\mathrm{cs}}^{m}\left(A_{0}, \hat{A}\right)$ in both cases of either $\operatorname{def} A_{0}=m<\infty$ or $\operatorname{def} A_{0}=\infty$ are described by the same formulas (3.16) and (3.17).

Remark 3.8. Let $A_{0}$ be defined by (3.32) or (3.33), and $F=\left(F_{1}, \ldots, F_{m}\right)$, where $F_{1}, \ldots, F_{m}$ are linearly independent elements of $R\left(A_{0}\right)^{\perp} \cap D(\hat{A})$. Then,

$$
\left(\widehat{A} F^{t}, F\right)=\mathbf{0} \Longleftrightarrow F_{i} \in D\left(A_{0}\right), \quad i=1, \ldots, m
$$

Let now the minimal symmetric operator $A_{0}$ be defined by

$$
A_{0} \subset \widehat{A}, \quad D\left(A_{0}\right)=\left\{x \in D(\hat{A}):\left(\widehat{A} x, F_{i}\right)=0\right\}, \quad F_{i} \in D\left(A_{0}\right),
$$

$i=1, \ldots, m$, and $F_{1}, \ldots, F_{m}$ are linearly independent elements of $D\left(A_{0}\right)$. Then from the above remark and Theorem 3.5 follows the next corollary, which describes the most "simple" extensions of $A_{0}$.

Corollary 3.9. (i) For every $B \in E_{\mathrm{cs}}^{m}\left(A_{0}, \widehat{A}\right)$, where $A_{0}$ satisfies (3.36), there exists a Hermitian $m \times m$ matrix $C$ with $\operatorname{det} C \neq 0$, such that (3.17) is fulfilled.

(ii) Conversely, for every Hermitian $m \times m$ matrix $C$, with rank $C=n \leq m$, the operator $B$ defined by (3.17) belongs to $E_{\mathrm{cs}}^{n}\left(A_{0}, \hat{A}\right)$.

The unique solution of (3.17) is given by (3.30).

The next theorem is useful for applications and gives the criterion of correctness of below problems and their solutions.

Theorem 3.10. Let

$$
B x=\widehat{A} x-(\widehat{A} F) C\left(\widehat{A} x, F^{t}\right)=f, \quad D(B)=D(\widehat{A}),
$$

where $\widehat{A}$ as in Lemma 3.1, $C$ a Hermitian $m \times m$ matrix with rank $C=n, F_{1}, \ldots, F_{m}$ linearly independent elements of $D(\hat{A})$. Then $B$ is correct and selfadjoint operator with $\operatorname{dim} R(B-$ $\widehat{A})=n$ if and only if

$$
\operatorname{det}\left[I-\overline{\left(\widehat{A} F^{t}, F\right)} C\right] \neq 0
$$

and the unique solution of (3.37) is given by

$$
x=B^{-1} f=\hat{A}^{-1} f+F C\left[I-\overline{\left(\widehat{A} F^{t}, F\right)} C\right]^{-1}\left(f, F^{t}\right) .
$$

Proof. We define corresponding to this problem the minimal operator $A_{0}$ as a restriction of $\widehat{A}$ by (3.32).

If $n=m$, then the theorem is true by Theorem 3.5.

While if $n<m$ and $B \in E_{\mathrm{cs}}^{n}\left(A_{0}, \widehat{A}\right)$, then from (3.37), we have $B x=f$ and

$$
\begin{aligned}
\left(F^{t}, f\right) & =\left(F^{t}, \widehat{A} x\right)-\left(F^{t}, \widehat{A} F\right) \overline{C\left(\hat{A} x, F^{t}\right)} \\
& =\left[I-\left(\widehat{A} F^{t}, F\right) \bar{C}\right]\left(F^{t}, \widehat{A} x\right)
\end{aligned}
$$


or

$$
\left[I-\overline{\left(\widehat{A} F^{t}, F\right)} C\right]\left(\widehat{A} x, F^{t}\right)=\left(f, F^{t}\right), \quad \forall f \in \mathbb{H} .
$$

Let $L=I-\overline{\left(\widehat{A} F^{t}, F\right)} C$ and $\operatorname{rank} L=k<m$. If we suppose that the first $k$ lines of the matrix $L$ are linearly independent, then for $f=\psi_{k+1}$, where $\left(F_{i}, \psi_{k}\right)=\delta_{i, k}, i, k=1, \ldots, m$, the system $L\left(\hat{A} x, F^{t}\right)=\left(f, F^{t}\right)$ has no solution, since the rank of the augmented matrix is $k+1 \neq k$. Then $B x=\psi_{k+1}$ has no solution and $R(B) \neq \mathbb{M}$. Consequently, $B$ is not a correct operator. So (3.38) holds true. Conversely, let $\operatorname{det} L \neq 0$, then by Theorem 3.5, we have that $B \in E_{\mathrm{cs}}^{n}\left(A_{0}, \widehat{A}\right)$.

We recall that a Hermitian $m \times m$ matrix $C=\left(c_{i j}\right)$ is called negative semidefinite (negative definite) if $\sum_{i=1}^{m} \sum_{j=1}^{m} \bar{\xi}_{i} \xi_{j} c_{i j} \leq 0$,

$$
\left(\sum_{i=1}^{m} \sum_{j=1}^{m} \bar{\xi}_{i} \xi_{j} c_{i j}<0\right), \quad \forall \xi=\left(\xi_{1}, \ldots, \xi_{m}\right) \in \mathbb{C}^{m}\left(\xi \in \mathbb{C}^{m} \backslash\{0\}\right)
$$

Theorem 3.11. If in Theorem $3.2 \hat{A}$ is positive operator and $C$ is negative semidefinite matrix, then $B$, defined by (3.17), is a positive operator.

Proof. We will show that $(B x, x) \geq 0$ for all $x \in D(B)$.

$$
\begin{aligned}
(B x, x) & =\left(\hat{A} x-(\widehat{A} F) C\left(\widehat{A} x, F^{t}\right), x\right)=(\widehat{A} x, x)-(\widehat{A} F, x) C\left(\widehat{A} x, F^{t}\right) \\
& =(\widehat{A} x, x)-\overline{(\widehat{A} x, F)} C\left(\widehat{A} x, F^{t}\right)=(\widehat{A} x, x)-\sum_{i=1}^{m} \sum_{j=1}^{m} \overline{\left(\widehat{A} x, F_{i}\right)}\left(\widehat{A} x, F_{j}\right) c_{i j} \geq 0,
\end{aligned}
$$

for $C$ is negative and semidefinite.

We remind that an operator $\widehat{A}: \mathbb{H} \rightarrow \mathbb{W}$ is called positive definite if there exists a positive real number $k$ such that

$$
(\widehat{A} x, x) \geq k\|x\|^{2}, \quad \forall x \in D(\widehat{A}) .
$$

Theorem 3.12. If the operator $\hat{A}$ in Theorem 3.2 is positive definite, then the operator $B$, which is defined by the relation (3.17), is positive definite whenever the matrix $C$ is Hermitian and satisfies the inequality

$$
k>\sum_{i=1}^{m} \sum_{j=1}^{m}\left\|\widehat{A} F_{i}\left|\left\||| \hat{A} F_{j}\right\|\right| c_{i j} \mid\right.
$$

and positive when $k \geq \sum_{i=1}^{m} \sum_{j=1}^{m}\left\|\hat{A} F_{i}\right\|\left\|\hat{A} F_{j}\right\|\left|c_{i j}\right|$. 
Proof. For $x \in D(B)$, we have

$$
\begin{aligned}
(B x, x) & =\left(\widehat{A} x-(\hat{A} F) C\left(\widehat{A} x, F^{t}\right), x\right)=(\widehat{A} x, x)-\overline{(x, \hat{A} F)} C\left(x, \widehat{A} F^{t}\right) \\
& \geq k\|x\|^{2}-\sum_{i=1}^{m} \sum_{j=1}^{m}\left|\overline{\left(x, \hat{A} F_{i}\right)}\left(x, \widehat{A} F_{j}\right) c_{i j}\right| \\
& \geq\left(k-\sum_{i=1}^{m} \sum_{j=1}^{m}\left\|\hat { A } F _ { i } \left|\left\|\left|\hat{A} F_{j} \|\right| c_{i j} \mid\right)\|x\|^{2} .\right.\right.\right.
\end{aligned}
$$

The theorem now easily follows.

Now we will state Theorem 3.2, in the following more general form, which is useful in the solutions of differential equations.

Theorem 3.13. Suppose that $A_{0}, \hat{A}$ are as in Theorem 3.2. Then the following hold.

(i) For every $B \in E_{\mathrm{cs}}^{m}\left(A_{0}, \hat{A}\right)$, there exists a vector $Q=\left(q_{1}, \ldots, q_{m}\right)$, where $q_{1}, \ldots, q_{m}$ are linearly independent elements from $D\left(A_{0}\right)^{\perp}$ and a Hermitian invertible $m \times m$ matrix $C$, such that

$$
\begin{gathered}
\operatorname{det}\left[I-\overline{\left(Q^{t}, \hat{A}^{-1} Q\right)} C\right] \neq 0, \\
B x=\widehat{A} x-Q C\left(x, Q^{t}\right)=f, \quad D(B)=D(\widehat{A}) .
\end{gathered}
$$

(ii) Conversely, for every vector $Q=\left(q_{1}, \ldots, q_{m}\right)$, defined as above, and Hermitian $m \times m$ matrix $C$, which has rank $C=n$ and satisfies (3.47), the operator $B$ defined by (3.48) belongs to $E_{\mathrm{cs}}^{n}\left(A_{0}, \hat{A}\right)$.

The unique solution of (3.48) is given by the formula

$$
x=\widehat{A}^{-1} f+\left(\widehat{A}^{-1} Q\right) C\left[I-\left(\overline{Q^{t}, \hat{A}^{-1} Q}\right) C\right]^{-1}\left(f, \hat{A}^{-1} Q^{t}\right)
$$

for all $f \in \mathbb{H}$.

The proof easily follows from Theorem 3.2 by substituting $Q=\widehat{A} F, F=\widehat{A}^{-1} Q$, where $Q=\left(q_{1}, \ldots, q_{m}\right), q_{i} \in D\left(A_{0}\right)^{\perp}, i=1, \ldots, m$.

Corollary 3.14. For every vector $Q=\left(q_{1}, \ldots, q_{m}\right)$, where $q_{1}, \ldots, q_{m}$ are linearly independent elements of $D\left(A_{0}\right)^{\perp} \cap R\left(A_{0}\right), i=1, \ldots, m$, and for every Hermitian $m \times m$ matrix $C$, with rank $C=n$, the operator $B$ defined by (3.48) belongs to $E_{\mathrm{cs}}^{n}\left(A_{0}, \hat{A}\right)$

The unique solution of (3.48) is given by the formula

$$
x=B^{-1} f=\widehat{A}^{-1} f+\left(\hat{A}^{-1} Q\right) C\left(f, \hat{A}^{-1} Q^{t}\right), \quad \forall f \in \mathbb{H} .
$$


Let now the minimal symmetric operator $A_{0}$ have finite defect and be defined by the relations

$$
A_{0} x=\widehat{A} x, \quad \forall x \in D\left(A_{0}\right), \quad D\left(A_{0}\right)=\left\{x \in D(\widehat{A}):\left(x, Q^{t}\right)=0\right\}
$$

where $Q$ is defined as in Theorem 3.13. Then $\operatorname{dim} D\left(A_{0}\right)^{\perp}=m$ and $\operatorname{def} A_{0}=\operatorname{dim} R\left(A_{0}\right)^{\perp}=$ $m$.

In this case, we restate Theorems $3.5,3.11$, and 3.12 in the following more general form.

Theorem 3.15. (a) For every $B \in E_{\mathrm{cs}}^{m}\left(A_{0}, \hat{A}\right)$, where $A_{0}$ is defined by (3.51), there exists a Hermitian $m \times m$ matrix $C$ with $\operatorname{det} C \neq 0$, such that (3.47) and (3.48) are fulfilled.

(b) Conversely, for every Hermitian $m \times m$ matrix $C$, which satisfies (3.47) and has rank $C=n$, the operator $B$ defined by (3.48) belongs to $E_{\mathrm{cs}}^{n}\left(A_{0}, \hat{A}\right)$. The unique solution of (3.48) is given by (3.49).

(c) If the operator $\hat{A}$ is positive and the matrix $C$ is negative semidefinite, then $B$ is positive.

(d) If $\hat{A}$ is positive definite (so it satisfies a relation (3.44)) and if $C$ is a Hermitian $m \times m$ matrix which satisfies the inequality

$$
k>\sum_{i=1}^{m} \sum_{j=1}^{m}\left\|q_{i}|\||\left|q_{j}\right||| c_{i j} \mid\right.
$$

then $B$ is positive definite; it is positive when $k \geq \sum_{i=1}^{m} \sum_{j=1}^{m}\left\|q_{i}\right\|\left\|q_{j}\right\|\left|c_{i j}\right|$,

Proof. Since $\hat{A}$ is selfadjoint and $R(\hat{A})=\mathbb{H}$, for every $x \in D(\hat{A})$, we have $\left(x, q_{i}\right)=$ $\left(x, \widehat{A} \widehat{A}^{-1} q_{i}\right)=\left(\hat{A} x, F_{i}\right)$, where $F_{i}=\widehat{A}^{-1} q_{i}, i=1,2, \ldots, m$.

It is clear that $F_{i} \in D(\hat{A}), i=1,2, \ldots, m$, and that they are linearly independent. If we substitute $Q=\widehat{A} F, Q^{t}=\widehat{A} F^{t}$ in (3.47), (3.48), and (3.49), then we receive the relations (3.16), (3.17), and (3.18) of Theorem 3.2, which hold true. Because of Theorems 3.11, 3.12 , and the relations $q_{i}=\hat{A} F_{i}, i=1,2, \ldots, m$, cases (c) and (d) of the present theorem are true.

Remark 3.16. Suppose that $A_{0}, \widehat{A}$ are as in Theorem 3.15 and $Q=\left(q_{1}, \ldots, q_{m}\right)$, where $q_{1}, \ldots, q_{m}$ are linearly independent elements of $D\left(A_{0}\right)^{\perp}$, then

$$
\begin{aligned}
& R\left(A_{0}\right)=\left\{f \in \mathbb{U}:\left(f, \hat{A}^{-1} q_{j}\right)=0, j=1, \ldots, m\right\}, \\
& \left(Q^{\prime}, \hat{A}^{-1} Q\right)=\mathbf{0} \Longleftrightarrow q_{i} \in R\left(A_{0}\right), \quad i=1, \ldots, m .
\end{aligned}
$$

Let now the minimal symmetric operator $A_{0}$ be defined by the relation

$$
A_{0} \subset \widehat{A}, \quad D\left(A_{0}\right)=\left\{x \in D(\hat{A}):\left(x, Q^{t}\right)=\mathbf{0}, Q \in R\left(A_{0}\right)^{m}\right\}
$$


and let $Q=\left(q_{1}, \ldots, q_{m}\right), q_{1}, \ldots, q_{m}$ be linearly independent elements of $D\left(A_{0}\right)^{\perp}$. By the above remark and Theorem 3.15, we take the following corollary, which describes the most "simple" extensions of $A_{0}$.

COROLlary 3.17. (i) For every $B \in E_{\mathrm{cs}}^{m}\left(A_{0}, \hat{A}\right)$, where $A_{0}$ satisfies (3.54), there exists a Hermitian $m \times m$ matrix $C$ with $\operatorname{det} C \neq 0$, such that (3.48) is fulfilled.

(ii) Conversely, for every Hermitian $m \times m$ matrix $C$, with rank $C=n \leq m$, the operator $B$ defined by (3.48) belongs to $E_{\mathrm{cs}}^{n}\left(A_{0}, \widehat{A}\right)$, where $A_{0}$ satisfies (3.54).

The unique solution of (3.48) is given by (3.50).

Let now $G=\left(g_{1}, \ldots, g_{m}\right)$, where $g_{1}, \ldots, g_{m}$ are arbitrary elements of $\mathbb{H}, \hat{A}$ as in Theorem 3.2, and $Q$ satisfies (3.51). Then holds the next corollary which is useful for applications.

Corollary 3.18. (i) If the operator $B: \mathbb{M} \rightarrow \mathbb{U}$ defined by

$$
B x=\widehat{A} x-G\left(x, Q^{t}\right)=f, \quad D(B)=D(\hat{A})
$$

is correct and selfadjoint and $\operatorname{dim} R(B-\hat{A})=m$, then the elements $q_{1}, \ldots, q_{m}$ are linearly independent and there exists a Hermitian, invertible $m \times m$ matrix $C$ such that $G=Q C$, where C satisfies (3.47).

(ii) Conversely, if there exists a Hermitian, $m \times m$ matrix $C$ such that $G=Q C$, where $C$ satisfies (3.47), then $B$ is correct and selfadjoint. If also $\operatorname{det} C \neq 0$, then $\operatorname{dim} R(B-\widehat{A})=m$.

Proof. Follows easily from Theorem 3.15 by defining a minimal operator $A_{0}$ by (3.51).

Let now $G=\left(g_{1}, \ldots, g_{n}\right), Q=\left(q_{1}, \ldots, q_{n}\right)$, where $g_{i} \in \mathbb{U}, q_{i} \in D\left(A_{0}\right)^{\perp} \subset \mathbb{H}, i=1, \ldots, n$. We suppose that the elements $q_{1}, \ldots, q_{m}(m<n)$ are linearly independent and for the rest $q_{m+1}, \ldots, q_{n}$, there exists an $(n-m) \times m$ matrix $M=\left(\mu_{i j}\right)$ such that $Q_{n-m}^{t}=M Q^{t m}$, where $Q^{m}=\left(q_{1}, \ldots, q_{m}\right), Q_{n-m}=\left(q_{m+1}, \ldots, q_{n}\right), Q^{t m}=\operatorname{col}\left(q_{1}, \ldots, q_{m}\right)$, and $Q_{n-m}^{t}=\operatorname{col}\left(q_{m+1}\right.$, $\left.\ldots, q_{n}\right)$.

A generalization of Theorem 3.13 is the following theorem.

Theorem 3.19. (i) If the operator $B: \mathbb{W} \rightarrow \mathbb{W}$ defined by (3.55) is correct and selfadjoint and $R(B-\hat{A})=m$, then the elements of the matrix $G^{m}+G_{n-m} \bar{M}$ are linearly independent and there exists a Hermitian invertible $m \times m$ matrix $C$ such that

$$
\begin{gathered}
G^{m}+G_{n-m} \bar{M}=Q^{m} C, \\
\operatorname{det}\left[I-\overline{\left(Q^{t m}, \hat{A}^{-1} Q^{m}\right)} C\right] \neq 0 .
\end{gathered}
$$

(ii) Conversely, if there exists a Hermitian $m \times m$ matrix $C$ such that relations (3.56) and (3.57) are satisfied, then $B$ is correct and selfadjoint. If also $\operatorname{det} C \neq 0$, then $\operatorname{dim} R(B-$ $\widehat{A})=m$.

The solution of problem (3.55) is given by the formula

$$
x=\hat{A}^{-1} f+\left(\hat{A}^{-1} Q^{m}\right) C\left[I-\overline{\left(Q^{t m}, \hat{A}^{-1} Q^{m}\right)} C\right]^{-1}\left(f, \hat{A}^{-1} Q^{t m}\right)
$$

for all $f \in \mathbb{H}$. 
Proof. We have

$$
\begin{aligned}
G\left(x, Q^{t}\right) & =G^{m}\left(x, Q^{t m}\right)+G_{n-m}\left(x, Q_{n-m}^{t}\right)=G^{m}\left(x, Q^{t m}\right)+G_{n-m}\left(x, M Q^{t m}\right) \\
& =G^{m}\left(x, Q^{t m}\right)+G_{n-m} \bar{M}\left(x, Q^{t m}\right)=\left(G^{m}+G_{n-m} \bar{M}\right)\left(x, Q^{t m}\right) .
\end{aligned}
$$

If we substitute the term $G\left(x, Q^{t}\right)$ in (3.55) with its equal from above, then we take

$$
B x=\widehat{A} x-\left(G^{m}+G_{n-m} \bar{M}\right)\left(x, Q^{t m}\right)=f .
$$

Now the operator $B$ in (3.60) has the form of the operator $B$ in (3.55), where instead of $G$ we have $G^{m}+G_{n-m} \bar{M}$ and instead of $Q$ we have $Q^{m}$. So according to Corollary 3.18, the relations (3.56) and (3.57) hold true; also (3.49) implies (3.58).

In the following examples, $\mathbb{T}^{1}(0,1)\left(\mathbb{T}^{2}(0,1)\right)$ denotes the Sobolev space of all complex functions of $L_{2}(0,1)$, which have generalized derivatives up to first-(second-)order, Lebesgue integrable.

\section{Examples}

Example 4.1. For every real number $c$, the operator $B: L_{2}(o, 1) \rightarrow L_{2}(0,1)$ corresponding to the problem

$$
\begin{gathered}
B u=i u^{\prime}-c x \int_{0}^{1} x u(x) d x=f(x), \\
D(B)=\left\{u \in \mathbb{H}^{1}(0,1): u(0)=-u(1)\right\}
\end{gathered}
$$

is a correct selfadjoint extension of the minimal symmetric operator $A_{0}$ defined by

$$
A_{0} \subset B, \quad D\left(A_{0}\right)=\left\{u \in D(B): \int_{0}^{1} x u(x) d x=0\right\}
$$

The unique solution of (4.1)-(4.2) is given by the formula

$$
u(x)=\frac{i}{2} \int_{0}^{1} f(t) d t-i \int_{0}^{x} f(t) d t+\frac{c i}{16}\left(1-2 x^{2}\right) \int_{0}^{1}\left(1-2 t^{2}\right) f(t) d t
$$

Proof. By comparing (4.1) with (3.48) and (4.3) with (3.54), we take

$$
\widehat{A} u=i u^{\prime}, \quad D(\hat{A})=D(B), \quad m=1, \quad C=c, \quad Q(x)=x, \quad x \in D\left(A_{0}\right)^{\perp} .
$$

It is evident that $A_{0}$ is minimal symmetric operator. From [6, page 272] (in our case $\theta=\pi$ ) follows that $\widehat{A}$ is selfadjoint and it is easily seen that

$$
\widehat{A}^{-1} f=\frac{i}{2} \int_{0}^{1} f(t) d t-i \int_{0}^{x} f(t) d t, \quad \forall f \in \mathbb{W}
$$


Then

$$
\begin{gathered}
\hat{A}^{-1} Q=\frac{i}{2} \int_{0}^{1} t d t-i \int_{0}^{x} t d t=\frac{i}{4}\left(1-2 x^{2}\right), \\
\left(Q^{\prime}, \hat{A}^{-1} Q\right)=\int_{0}^{1} t \overline{\frac{i}{4}\left(1-2 t^{2}\right)} d t=0 \\
\left(f, \hat{A}^{-1} Q\right)=\int_{0}^{1} f(t) \overline{\frac{i}{4}\left(1-2 t^{2}\right)} d t=-\frac{i}{4} \int_{0}^{1}\left(1-2 t^{2}\right) f(t) d t .
\end{gathered}
$$

The condition $\left(Q^{\prime}, \hat{A}^{-1} Q\right)=0$ and Remark 3.16 imply that $Q \in R\left(A_{0}\right)$ and from Corollary 3.17 follows the validity of this example.

Example 4.2. The operator $\widehat{A}: L_{2}(0,1) \rightarrow L_{2}(0,1)$ defined by

$$
\begin{gathered}
\hat{A} u=-u^{\prime \prime}=f, \\
D(\hat{A})=\left\{u \in \mathbb{T}^{2}(0,1): u(0)=-u(1), u^{\prime}(0)=-u^{\prime}(1)\right\}
\end{gathered}
$$

is a correct, selfadjoint, positive definite operator and satisfies the inequality

$$
(\hat{A} u, u)=\int_{0}^{1}\left|u^{\prime}\right|^{2} d x \geq 4 \int_{0}^{1}|u|^{2} d x
$$

For every $f \in L_{2}(0,1)$, the unique solution $u$ of the problem (4.8)-(4.9) is given by the formula

$$
u=\hat{A}^{-1} f=-\int_{0}^{t}(t-\xi) f(\xi) d \xi+\frac{t}{2} \int_{0}^{1} f(\xi) d \xi-\frac{1}{2} \int_{0}^{1}\left(\xi-\frac{1}{2}\right) f(\xi) d \xi
$$

Proof. Indeed, formula (4.11) is found by two direct integrations of (4.8), where (4.9) is taken into consideration. That $\hat{A}^{-1}$ is continuous is proved easily by showing, using Schwarz's inequality and formula (4.11), that $\hat{A}^{-1}$ is a bounded operator. Hence $\hat{A}$ is a correct operator. We show that $\hat{A}$ is selfadjoint. From formula (4.11), we take

$$
\begin{aligned}
\hat{A}^{-1} f= & \int_{0}^{t}(\xi-t) f(\xi) d \xi+\int_{0}^{t}\left[\frac{t}{2}-\frac{1}{2}\left(\xi-\frac{1}{2}\right)\right] f(\xi) d \xi \\
& +\int_{t}^{1}\left[\frac{t}{2}-\frac{1}{2}\left(\xi-\frac{1}{2}\right)\right] f(\xi) d \xi \\
= & \int_{0}^{1}\left[\frac{1}{2}\left(\xi-t+\frac{1}{2}\right) \eta(t-\xi)+\frac{1}{2}\left(t-\xi+\frac{1}{2}\right) \eta(\xi-t)\right] f(\xi) d \xi
\end{aligned}
$$


So, the integral Kernel of $\hat{A}^{-1}$ is the function

$$
K(t, \xi)=\frac{1}{2}\left(\xi-t+\frac{1}{2}\right) \eta(t-\xi)+\frac{1}{2}\left(t-\xi+\frac{1}{2}\right) \eta(\xi-t),
$$

where

$$
\eta(x)=\left\{\begin{array}{ll}
1, & x>0, \\
0, & x \leq 0,
\end{array}\right. \text { is the Heaviside's function. }
$$

Since

$$
\overline{K(t, \xi)}=\frac{1}{2}\left(t-\xi+\frac{1}{2}\right) \eta(\xi-t)+\frac{1}{2}\left(\xi-t+\frac{1}{2}\right) \eta(t-\xi)=K(\xi, t)
$$

it follows from [16] that $\hat{A}^{-1}$ is selfadjoint. Then, from the equalities $\hat{A}^{-1}=\left(\hat{A}^{-1}\right)^{*}=$ $\left(\widehat{A}^{*}\right)^{-1}[11]$ follows that $D(\widehat{A})=D\left(\hat{A}^{*}\right)$. On the other hand, for all $x, y \in D(\widehat{A})=D\left(\hat{A}^{*}\right)$, we have

$$
(\widehat{A} x, y)=\left(\hat{A} x, \hat{A}^{-1} \hat{A} y\right)=(x, \widehat{A} y)
$$

The above two remarks imply that $\hat{A}=\hat{A}^{*}$.

Next, we prove inequality (4.10), showing at the same time that $\hat{A}$ is positive definite. Let $u(x) \in D(\widehat{A})$. Since $u(0)=-u(1)$, we have

$$
u(x)=\int_{0}^{x} u^{\prime}(t) d t-u(1), \quad u(1)=\frac{1}{2} \int_{0}^{1} u^{\prime}(t) d t
$$

From these equalities, we take

$$
u(x)=\int_{0}^{1} u^{\prime}(t) \eta(x-t) d t-\frac{1}{2} \int_{0}^{1} u^{\prime}(t) d t=\int_{0}^{1} u^{\prime}(t)\left[\eta(x-t)-\frac{1}{2}\right] d t
$$

and then $|u(x)| \leq 1 / 2 \int_{0}^{1}\left|u^{\prime}(t)\right| d t$. Using Schwarz's inequality, we take

$$
|u(x)|^{2} \leq \frac{1}{4}\left(\int_{0}^{1}\left|u^{\prime}(t)\right| d t\right)^{2} \leq \frac{1}{4} \int_{0}^{1}\left|u^{\prime}(t)\right|^{2} d t .
$$

Then

$$
\int_{0}^{1}|u(x)|^{2} d x \leq \frac{1}{4} \int_{0}^{1} d x \int_{0}^{1}\left|u^{\prime}(t)\right|^{2} d t=\frac{1}{4} \int_{0}^{1}\left|u^{\prime}(t)\right|^{2} d t
$$


Now, by (4.9), we have

$$
\begin{aligned}
(\widehat{A} u, u) & =-\int_{0}^{1} u^{\prime \prime} \bar{u} d x=-\left.u^{\prime}(x) \bar{u}(x)\right|_{0} ^{1}+\int_{0}^{1} u^{\prime} \bar{u}^{\prime} d x \\
& =-u^{\prime}(1) \bar{u}(1)+u^{\prime}(0) \bar{u}(0)+\int_{0}^{1}\left|u^{\prime}\right|^{2} d x=\int_{0}^{1}\left|u^{\prime}(x)\right|^{2} d x . \\
& \geq 4 \int_{0}^{1}|u(x)|^{2} d x .
\end{aligned}
$$

Example 4.3. The operator $B: L_{2}(0,1) \rightarrow L_{2}(0,1)$, which corresponds to the problem

$$
B u=-u^{\prime \prime}-c\left(3 t^{2}-1\right) \int_{0}^{1}\left(2 t^{4}-4 t^{2}+1\right) u^{\prime \prime}(t) d t=f(t)
$$

with

$$
D(B)=\left\{u \in \mathbb{H}^{2}(0,1): u(0)=-u(1), u^{\prime}(0)=-u^{\prime}(1)\right\}
$$

and $c$ a constant is

(i) correct and selfadjoint if and only if $c$ is real and $c \neq 105 / 64$,

(ii) positive definite when $c$ is real and $|c|<5 / 8$ and positive when $c$ is real and $c \leq 5 / 8$. The unique solution of problem (4.22) for every $f \in L_{2}(0,1)$ is given by

$$
u(t)=\hat{A}^{-1} f+\left(2 t^{4}-4 t^{2}+1\right) \frac{105 c}{8(105-64 c)} \int_{0}^{1}\left(2 t^{4}-4 t^{2}+1\right) f(t) d t
$$

where $\hat{A}^{-1} f$ is found by (4.11) of Example 4.2.

Proof. If we compare (4.22) with (3.37), it is natural to take $\hat{A} u=-u^{\prime \prime}$ with $D(\hat{A})=D(B)$, $m=1, F(t)=2 t^{4}-4 t^{2}+1$. We easily see that $F \in D(\hat{A})$ and $\hat{A} F=-8\left(3 t^{2}-1\right)$. Then (4.22) can be written as follows:

$$
B u=-u^{\prime \prime}-\frac{c}{8}(-8)\left(3 t^{2}-1\right) \int_{0}^{1}\left(2 t^{4}-4 t^{2}+1\right)\left(-u^{\prime \prime}(t)\right) d t
$$

which is the form of (3.37) with $C=c / 8$. Then we find

$$
\begin{gathered}
\|\hat{A} F\|^{2}=64 \int_{0}^{1}\left(3 t^{2}-1\right)^{2} d t=\frac{256}{5}, \\
(\widehat{A} F, F)=-8 \int_{0}^{1}\left(3 t^{2}-1\right)\left(2 t^{4}-4 t^{2}+1\right) d t=\frac{512}{105}, \\
\operatorname{det}\left[I-\overline{\left(\widehat{A} F^{t}, F\right)} C\right]=1-\frac{512}{105} \frac{c}{8}=\frac{105-64 c}{105} .
\end{gathered}
$$

Relation (4.10) shows that $k=4$. From Theorem 3.10, we conclude that $B$ is correct and selfadjoint operator if and only if $c \in \mathbb{R}$ and $(105-64 c) / 105 \neq 0$, or $c \neq 105 / 64$. 
To find the values of $c \in \mathbb{R}$ for which $B$ is positive definite, we use (3.45)

$$
4=k>\|\hat{A} F\|^{2} \frac{|c|}{8}=\frac{|c|}{8} \frac{256}{5} \quad \text { or } \quad|c|<\frac{5}{8}
$$

So for $c \in \mathbb{R}$ and $|c|<5 / 8$, the operator $B$ is positive definite.

By Theorem 3.12 for $|c| \leq 5 / 8, B$ is positive. Also by Theorem 3.11 for $c \leq 0, B$ is again positive. So we conclude that for $c \leq 5 / 8, B$ is a positive operator.

Example 4.4. The operator $B: L_{2}(0,1) \rightarrow L_{2}(0,1)$, which corresponds to the problem

$$
\begin{gathered}
B u=-u^{\prime \prime}+c_{1} \int_{0}^{1}\left(t^{2}-t\right) u^{\prime \prime}(t) d t+c_{2} \sin (\pi x) \int_{0}^{1} \sin (\pi t) u^{\prime \prime}(t) d t=f(x), \\
D(B)=\left\{u \in \mathbb{T}^{2}(0,1): u(0)=-u(1), u^{\prime}(0)=-u^{\prime}(1)\right\}
\end{gathered}
$$

is

(i) correct and selfadjoint if and only if $c_{1}, c_{2}$ are real constants such that

$$
\left(1-\frac{c_{1}}{6}\right)\left(1+\frac{c_{2}}{2}\right)+\frac{8 c_{1} c_{2}}{\pi^{4}} \neq 0
$$

(ii) positive definite when $c_{1}, c_{2}$ are reals and satisfy the inequality

$$
4\left|c_{1}\right|+\pi^{2}\left|c_{2}\right|<8
$$

(iii) positive when $c_{1} \leq 0, c_{2} \geq 0$ or when $4\left|c_{1}\right|+\pi^{2}\left|c_{2}\right| \leq 8$.

Proof. By comparing again (4.28) with (3.37), we take $\hat{A} u=-u^{\prime \prime}$ with $D(\hat{A})=D(B)$, $m=2, F_{1}(t)=t^{2}-t, F_{2}(t)=\sin (\pi t)$, and $C=\left(\begin{array}{cc}c_{1} / 2 & 0 \\ 0 & -c_{2} / \pi^{2}\end{array}\right)$. Then we find $\hat{A} F_{1}=-2, \widehat{A} F_{2}=$ $\pi^{2} \sin (\pi t),\left(\widehat{A} F^{t}, F\right)=\left(\begin{array}{cc}1 / 3 & -4 / \pi \\ -4 / \pi & \pi^{2} / 2\end{array}\right)$. We notice that $C$ is Hermitian if and only if $c_{1}, c_{2} \in \mathbb{R}$. The condition (3.38) gives the relation (4.30). So $B$ is correct and selfadjoint operator if and only if $c_{1}, c_{2} \in \mathbb{R}$ and satisfy (4.30). By simple calculation, we find $\left\|\widehat{A} F_{1}\right\|^{2}=4$ and $\left\|\hat{A} F_{2}\right\|^{2}=\pi^{4} / 2$. From (3.45), it is implied that $B$ is positive definite if $c_{1}, c_{2} \in \mathbb{R}$ and satisfy inequality (4.31). By Theorem $3.11, B$ is positive if $C$ is negative semidefinite, that is, $c_{1} \leq 0, c_{2} \geq 0$. Also, by Theorem $3.12, B$ is positive if $c_{1}, c_{2}$ satisfy $4\left|c_{1}\right|+\pi^{2}\left|c_{2}\right| \leq 8$.

Example 4.5. The operator $B: L_{2}(0,1) \rightarrow L_{2}(0,1)$, with $D(B)$ defined by $(4.29)$ and $B$ by the equation

$$
B u=-u^{\prime \prime}-c \int_{0}^{1} u(t) d t=f(t)
$$

is

(i) correct and selfadjoint if and only if $c$ is a real number such that $c \neq 12$,

(ii) positive definite when $c$ is real and $|c|<4$ and positive when $c$ is real and $c \leq 4$. 
The unique solution of problem (4.32) is given by the formula

$$
\begin{aligned}
u(t)= & -\int_{0}^{t}(t-\xi) f(\xi) d \xi+\frac{t}{2} \int_{0}^{1} f(\xi) d \xi \\
& -\frac{1}{2} \int_{0}^{1}\left(\xi-\frac{1}{2}\right) f(\xi) d \xi+\frac{3 c\left(t^{2}-t\right)}{12-c} \int_{0}^{1}\left(\xi^{2}-\xi\right) f(\xi) d \xi .
\end{aligned}
$$

Proof. We refer now to Theorem 3.15. By comparing (4.32) with (3.48), we take $\hat{A} u=$ $-u^{\prime \prime}$ with $D(\hat{A})=D(B)$ and

$$
A_{0} \subset \widehat{A}, \quad \text { with } D\left(A_{0}\right)=\left\{u \in D(\widehat{A}): \int_{0}^{1} u(t) d t=0\right\} .
$$

Since $\hat{A}$ is a correct and selfadjoint operator, $A_{0}$ is a minimal symmetric operator. We take also $Q=1, C=c$. By Theorem $3.15, B$ is correct and selfadjoint if and only if $c \in \mathbb{R}$ and relation (3.47) is satisfied, that is, if

$$
1-c \int_{0}^{1}\left(\hat{A}^{-1} Q\right)(t) d t \neq 0
$$

where by (4.11),

$$
\begin{aligned}
\widehat{A}^{-1} Q=\widehat{A}^{-1} 1=- & \int_{0}^{t}(t-\xi) d \xi+\frac{t}{2} \int_{0}^{1} d \xi-\frac{1}{2} \int_{0}^{1}\left(\xi-\frac{1}{2}\right) d \xi=-\frac{1}{2}\left(t^{2}-t\right), \\
& \int_{0}^{1}\left(\hat{A}^{-1} Q\right)(t) d t=-\frac{1}{2} \int_{0}^{1}\left(t^{2}-t\right) d t=\frac{1}{12} .
\end{aligned}
$$

So, (4.35) implies that $(12-c) / 12 \neq 0$, that is, $c \neq 12$. By Theorem 3.15 , if $c \in \mathbb{R}$ and $|c|<4$, then $B$ is positive definite. Again by Theorem 3.15, if $c \leq 0$ and $|c| \leq 4$, that is, $c \leq 4$, then $B$ is a positive operator. The solution of the problem (4.32) is found by formula (3.49).

Let $\Omega=\left\{x \in \mathbb{R}^{2}:|x|<1\right\}, \partial \Omega=\gamma$, and $\mathbb{H}^{2}(\Omega)$ - the Sobolev space of all functions of $L_{2}(\Omega)$ which have their partial generalized derivatives up to second-order, Lebesgue integrable. The problem

$$
-\triangle u=f, \quad u_{\mid \gamma}=0, u \in \mathbb{H}^{2}(\Omega), f \in L_{2}(\Omega)
$$

is the well-known Dirichlet problem and it is known that the corresponding operator $\hat{A}$

(i) is correct and selfadjoint, and

$$
u=\widehat{A}^{-1} f=\int_{\Omega} G(x, y) f(y) d y, \quad \forall f \in L_{2}(\Omega),
$$

where $G(x, y)$ is Green's function,

(ii) is positive definite and

$$
(\hat{A} u, u)=\int_{\Omega}|\nabla u|^{2} d x \geq \frac{\pi^{2}}{2} \int_{\Omega}|u|^{2} d x=\frac{\pi^{2}}{2}\|u\|_{L_{2}(\Omega)}^{2} .
$$


This inequality which has been proved in [15, pages 194, 195] for real functions $u \in$ $C^{2}(\bar{\Omega}): u_{\mid \gamma}=0$ holds true for all $u \in \mathbb{M}^{2}(\Omega): u_{\mid \gamma}=0$, since $C^{2}(\bar{\Omega})$ is dense in $\mathbb{H}^{2}(\Omega)$ and for all $u \in D(\widehat{A})$, exist the real functions $g, h \in D(\hat{A})$ such that $u=g+i h,(\hat{A} u, u)=$ $(\hat{A} g, g)-i(\hat{A} g, h)+i(\hat{A} h, g)+(\hat{A} h, h) \geq\left(\pi^{2} / 2\right)\|g\|^{2}+\left(\pi^{2} / 2\right)\|h\|^{2}=\left(\pi^{2} / 2\right)\|u\|^{2}$ and since

$$
\begin{gathered}
(\hat{A} h, g)=-\int_{\Omega} g \Delta h d x=\int_{\Omega} \nabla g \nabla h d x, \\
(\hat{A} g, h)=-\int_{\Omega} h \triangle g d x=\int_{\Omega} \nabla g \nabla h d x, \quad g_{\mid \gamma}=h_{\mid \gamma}=0,(g, h)=(h, g), \\
\|u\|^{2}=(u, u)=(g+i h, g+i h)=(g, g)+(h, h)=\|g\|^{2}+\|h\|^{2} .
\end{gathered}
$$

Example 4.6. The operator $B: L_{2}(\Omega) \rightarrow L_{2}(\Omega)$ which corresponds to the problem

$$
\begin{gathered}
B u=-\triangle u-c \triangle v \int_{\Omega} u \triangle \bar{v} d x=f(x) \\
D(B)=\left\{u \in \mathbb{T}^{2}(\Omega): u_{\mid \gamma}=0\right\},
\end{gathered}
$$

where $v \in D(B), v \neq 0$, is correct and selfadjoint if and only if $1-c \int_{\Omega}|\nabla v|^{2} d x \neq 0$ and $B$ is positive definite if $|c|<\pi^{2} /\left(2 \int_{\Omega}|\Delta v|^{2} d x\right), B$ is positive if $c \leq \pi^{2} /\left(2 \int_{\Omega}|\triangle v|^{2} d x\right)$.

The unique solution of (4.42)-(4.43) is given by the formula

$$
u(x)=\int_{\Omega} G(x, y) f(y) d y+\frac{c v(x)}{1-c \int_{\Omega}|\nabla v|^{2} d y} \int_{\Omega} f(y) \overline{v(y)} d y
$$

Proof. From Green's formula, since $u_{\mid \gamma}=0, v_{\mid \gamma}=0$, we have

$$
\int_{\Omega}(\bar{v} \triangle u-u \triangle \bar{v}) d x=\int_{\gamma}\left(\bar{v} \frac{\partial u}{\partial n}-u \frac{\partial \bar{v}}{\partial n}\right) d s=0 .
$$

So,

$$
\int_{\Omega} \bar{v} \triangle u d x=\int_{\Omega} u \triangle \bar{v} d x
$$

Now (4.42) takes the form

$$
B u=-\triangle u-c \Delta v \int_{\Omega} \bar{v} \Delta u d x=f, \quad u \in D(B) .
$$

We refer now to Theorem 3.10 and take $m=1, F=v, \hat{A} u=-\triangle u$ with $D(\hat{A})=D(B)$. Then, since $v \neq 0$, we have $\|\hat{A} F\|^{2}=\int_{\Omega}|\triangle v|^{2} d x \neq 0$,

$$
\left(\widehat{A} F^{t}, F\right)=(\hat{A} v, v)=-\int_{\Omega} \bar{v} \Delta v d x=\int_{\Omega}|\nabla v|^{2} d x
$$


The last equality follows from Green's formula

$$
\int_{\Omega} u \Delta v d x=\int_{\gamma} u \frac{\partial v}{\partial n} d s-\int_{\Omega} \nabla v \nabla u d x
$$

by taking $u=\bar{v} \in D(B)$ and the fact that $v_{\mid \gamma}=0$.

Now, by Theorem $3.10, B$ is correct and selfadjoint if and only if $c \in \mathbb{R}$ and

$$
\operatorname{det}\left[I-\overline{\left(\widehat{A} F^{t}, F\right)} C\right]=1-c \int_{\Omega}|\nabla v|^{2} d x \neq 0
$$

By relations (3.39) and (4.39), we find (4.44) — the unique solution of problem (4.42)(4.43).

From (4.40) and Theorems 3.11, 3.12, it is implied that $k=\pi^{2} / 2$ and $B$ is positive definite if $|c|<\pi^{2} /\left(2 \int_{\Omega}|\Delta v|^{2} d x\right)$, and $B$ is positive if $c \leq \pi^{2} /\left(2 \int_{\Omega}|\Delta v|^{2} d x\right)$.

Let $\hat{A}$ be as above, $\lambda_{1}, \lambda_{2}$ its two eigenvalues and $v_{1}, v_{2}$ the eigenvectors of $\hat{A}$, corresponding to $\lambda_{1}, \lambda_{2}$. It is known that $v_{1}, v_{2}$ are linearly independent elements of $D(\hat{A})$, $\left(v_{1}, v_{2}\right)=0$, and $\lambda_{1}, \lambda_{2} \geq 0$. Let $\lambda_{1}, \lambda_{2}>0$.

Example 4.7. The operator $B: L_{2}(\Omega) \rightarrow L_{2}(\Omega)$ which corresponds to the problem

$$
\begin{gathered}
B u=-\triangle u-c_{1} v_{1} \int_{\Omega} \overline{v_{1}} \Delta u d x-c_{2} v_{2} \int_{\Omega} \overline{v_{2}} \Delta u d x=f(x), \\
D(B)=\left\{u \in \mathbb{T}^{2}(\Omega): u_{\mid \gamma}=0\right\}
\end{gathered}
$$

is

(i) correct and selfadjoint if and only if $c_{1}, c_{2}$ are real numbers such that

$$
\left(1+c_{1}\left\|v_{1}\right\|^{2}\right)\left(1+c_{2}\left\|v_{2}\right\|^{2}\right) \neq 0
$$

(ii) positive definite if $c_{1}, c_{2} \in \mathbb{R}$ and

$$
2 \lambda_{1}\left|c_{1}\right|\left\|v_{1}\right\|^{2}+2 \lambda_{2}\left|c_{2}\right|\left\|v_{2}\right\|^{2}<\pi^{2}
$$

(iii) positive if $c_{1}, c_{2} \in \mathbb{R}$ and

$$
c_{1}, c_{2} \leq 0 \text { or when } 2 \lambda_{1}\left|c_{1}\right|\left\|v_{1}\right\|^{2}+2 \lambda_{2}\left|c_{2}\right|\left\|v_{2}\right\|^{2} \leq \pi^{2}
$$

The unique solution of (4.51)-(4.52) is given by the formula

$$
u(x)=\int_{\Omega} G(x, y) f(y) d y+\sum_{i=1}^{2} \frac{c_{i} v_{i}(x)}{\lambda_{i}\left(1+c_{i}\left\|v_{i}\right\|^{2}\right)} \int_{\Omega} f(y) \overline{v_{i}(y)} d y
$$


Proof. Since $v_{i}=-\triangle v_{i} / \lambda_{i}, i=1,2$, from (4.51), we have

$$
B u=-\triangle u-\sum_{i=1}^{2} \frac{c_{i}}{\lambda_{i}}\left(-\triangle v_{i}\right) \int_{\Omega} \bar{v}_{i}(-\triangle u) d x=f(x) .
$$

By comparing (4.57) with (3.37), we take $\hat{A} u=-\triangle u, D(\hat{A})=D(B), m=2, F=$ $\left(v_{1}(x) v_{2}(x)\right), C=\left(\begin{array}{cc}c_{1} / \lambda_{1} & 0 \\ 0 & c_{2} / \lambda_{2}\end{array}\right)$. Then $\widehat{A} F^{t}=\left(\begin{array}{c}-\triangle v_{1} \\ -\triangle v_{2}\end{array}\right),\left(\hat{A} F^{t}, F\right)=-\left(\begin{array}{cc}\lambda_{1}\left\|v_{1}\right\|^{2} & 0 \\ 0 & \lambda_{2}\left\|v_{2}\right\|^{2}\end{array}\right),\left(f, F^{t}\right)=$ $\left(\begin{array}{l}\left(f, v_{1}\right) \\ \left(f, v_{2}\right)\end{array}\right), \operatorname{det}\left(I-\overline{\left(\hat{A} F^{t}, F\right)} C\right)=\left(1+c_{1}\left\|v_{1}\right\|^{2}\right)\left(1+c_{2}\left\|v_{2}\right\|^{2}\right) \neq 0$, that is, we received (4.53). Also, $\left\|\widehat{A} F_{i}\right\|=\left\|-\triangle v_{i}\right\|=\lambda_{i}\left\|v_{i}\right\|, i=1,2$ and

$$
\left(I-\overline{\left(\hat{A} F^{t}, F\right)} C\right)^{-1}=\left(\begin{array}{cc}
\frac{1}{\left(1+c_{1}\left\|v_{1}\right\|^{2}\right)} & 0 \\
0 & \frac{1}{\left(1+c_{2}\left\|v_{2}\right\|^{2}\right)}
\end{array}\right)
$$

From Theorem 3.12 and (4.40), it follows that $k=\pi^{2} / 2$ and operator $B$ is positive definite if $c_{1}, c_{2} \in \mathbb{R}$ and satisfy the inequality (4.54). By Theorem 3.11, the operator $B$ is positive if $C$ is negative semidefinite, that is, $c_{1}, c_{2} \leq 0$. Also, by Theorem $3.12, B$ is positive if $c_{1}, c_{2} \in \mathbb{R}$ and satisfy $2 \lambda_{1}\left|c_{1}\right|\left\|v_{1}\right\|^{2}+2 \lambda_{2}\left|c_{2}\right|\left\|v_{2}\right\|^{2} \leq \pi^{2}$.

The relations (3.39) and (4.39) give us the unique solution (4.56) of this problem.

The results of this paper can be applied to Hermitian matrices, which are the matrices of Hermitian operators in unitary spaces with respect to any orthonormal basis of the space.

Example 4.8. Let $\hat{A}$ be a Hermitian operator in the $n$-dimensional unitary space $E$, $\lambda_{1}, \ldots, \lambda_{m}$ its eigenvalues, which are real numbers, different from zero, with multiplicity $p_{1}, \ldots, p_{m}$ and $E_{1}, \ldots, E_{m}$, the corresponding eigenspaces. If we consider that $E$ endowed with an orthonormal basis $D$ consisted of eigenvectors of $\hat{A}$, such that the first $p_{1}$ elements of $D$ constitute a basis of $E_{1}$, the next $p_{2}$ elements constitute a basis of $E_{2}$ and so on, then the matrix of $\hat{A}$, with respect to this basis, is the following:

$$
\left(\begin{array}{ccccccc}
\lambda_{1} & & & & & & \\
& \ddots & & & & & \\
& & \lambda_{1} & & & & \\
& & & \ddots & & & \\
& & & & \lambda_{m} & & \\
& & & & & \ddots & \\
& & & & & & \lambda_{m}
\end{array}\right) \text {, }
$$

where all the other elements are zero. Let $A_{0}$ be the restriction of $\hat{A}$ onto the subspace $E_{1} \oplus \cdots \oplus E_{m-1}$, which is a symmetric operator on this subspace. Let also $\varepsilon_{1}, \ldots, \varepsilon_{p}$ be an orthonormal basis of $E_{m}$, where $p_{m}=p$. If we write $F=\left(\varepsilon_{1} \cdots \varepsilon_{p}\right)$, then Theorem 3.10 asserts that any invertible Hermitian extension $B$ of $A_{0}$ to the whole space $E$ which takes 
different values on $E_{m}$ than $\hat{A}$ is given by the formula

$$
B x=\widehat{A} x-(\hat{A} F) C\left(\widehat{A} x, F^{t}\right), \quad x \in E,
$$

where $C$ is an invertible Hermitian matrix which satisfies relation (3.16), that is,

$$
\operatorname{det}\left[I-\overline{\left(\widehat{A} F^{t}, F\right)} C\right] \neq 0 \text {. }
$$

This relation is equivalent to

$$
\operatorname{det}\left[C-\frac{1}{\lambda_{m}} I\right] \neq 0,
$$

which means that the number $1 / \lambda_{m}$ is not an eigenvalue of $C$. The action of $B$ on $E_{m}$ is found easily, which is described by the formula

$$
B x=\lambda_{m} x-\lambda_{m}^{2}\left(\varepsilon_{1} \cdots \varepsilon_{p}\right)\left(\begin{array}{c}
c_{11} \cdots c_{1 p} \\
\cdots \cdots \\
c_{p 1} \cdots c_{p p}
\end{array}\right)\left(\begin{array}{c}
x_{1} \\
\vdots \\
x_{p}
\end{array}\right),
$$

where $x=x_{1} \varepsilon_{1}+\cdots+x_{p} \varepsilon_{p} \in E_{m}$, and its matrix, with respect to $D$, is

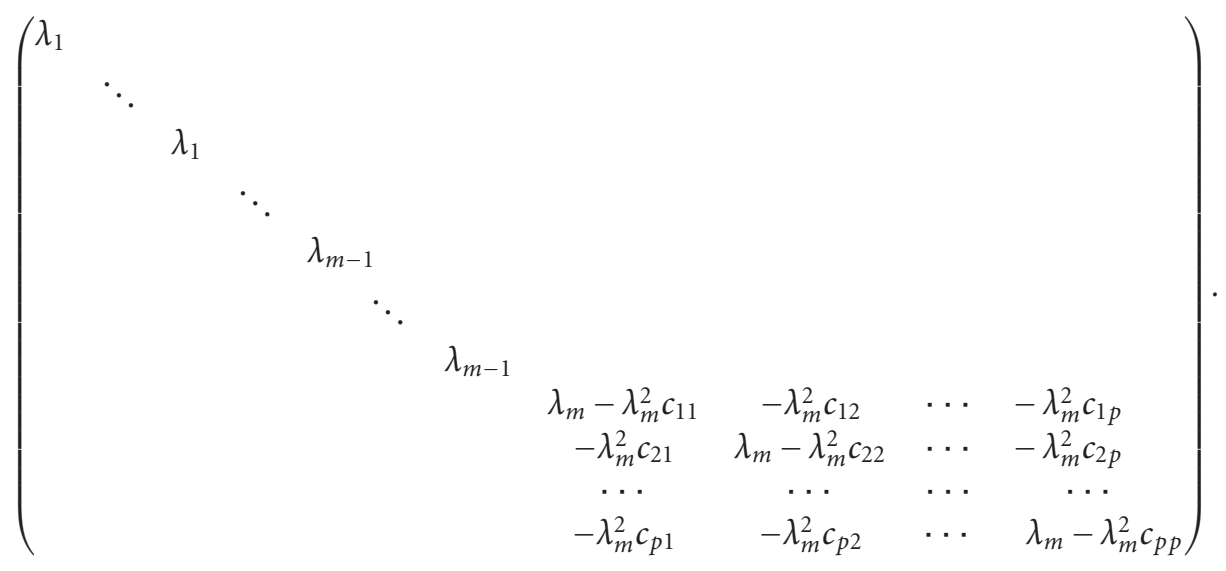

The $p \times p$ submatrix at the right-bottom place of the previous matrix represents an invertible Hermitian matrix which has not the number $\lambda_{m}$ as eigenvalue, and this is something expected.

Now, if $\hat{A}$ is positive definite, then if we choose the elements $c_{i j}$ of the matrix $C$ to satisfy the inequality

$$
k>\lambda_{m}^{2} \sum_{j=1}^{p} \sum_{i=1}^{p}\left|c_{i j}\right|,
$$

we obtain, by Theorem 3.12, a positive definite matrix $B$. 


\section{References}

[1] E. A. Coddington, Self-adjoint subspace extensions of nondensely defined symmetric operators, Advances in Math. 14 (1974), 309-332.

[2] E. A. Coddington and A. Dijksma, Self-adjoint subspaces and eigenfunction expansions for ordinary differential subspaces, J. Differential Equations 20 (1976), no. 2, 473-526.

[3] A. A. Dezin, General questions of the theory of boundary value problems, Nauka, Moscow, 1980.

[4] K. O. Friedrichs, Symmetric positive linear differential equations, Comm. Pure Appl. Math. 11 (1958), 333-418.

[5] V. I. Gorbachuk and M. L. Gorbachuk, Boundary value problems for operator differential equations, Mathematics and Its Applications (Soviet Series), vol. 48, Kluwer Academic, Dordrecht, 1991.

[6] T. Kato, Perturbation Theory for Linear Operators, Springer, Berlin, 1980.

[7] A. N. Kočubě̆, Extensions of a nondensely defined symmetric operator, Sibirsk. Mat. Ž 18 (1977), no. 2, 314-320, 478.

[8] _ Extensions of positive definite symmetric operators, Dokl. Akad. Nauk Ukrain. SSR Ser. A (1979), no. 3, 168-171, 237 (Russian).

[9] Symmetric operators and nonclassical spectral problems, Math. Notes 25 (1979), 224 229, (English translation).

[10] B. K. Kokebaev, M. Otelbaev, and A. N. Shinibekov, About Restrictions and Extensions of operators, Dokl. Akad. Nauk (D.A.N) SSSR 271 (1983), no. 6, 1307-1310 (Russian).

[11] E. Kreyszig, Introductory Functional Analysis with Applications, John Wiley \& Sons, New York, 1989.

[12] V. A. Mikhălets, Spectra of operators and boundary value problems, Spectral Analysis of Differential Operators, Akademiya Nauk Ukrainy SSR, Institut Matematiki, Kiev, 1980, pp. $106-131$.

[13] J. von Neumann, Allgemeine Eigenwerttheorie Hermitescher Funktionaloperatoren, Math. Ann. 102 (1929), 49-131 (German).

[14] R. Oinnarov and I. N. Parasidi, Correctly solvable extensions of operators with finite deficiencies in a Banach space, Izv. Akad. Nauk Kazah. SSR Ser. Fiz.-Mat. 144 (1988), no. 5, 42-46 (Russian).

[15] K. Rektorys, Variational Methods in Mathematical Physics and Engineering, "Mir", Moscow, 1985.

[16] I. Stakgold, Green's Functions and Boundary Value Problems, Pure and Applied Mathematics, John Wiley \& Sons, New York, 1979.

[17] M. I. Vishik, About of boundary value problems for elliptical differential equations, Mosk. Math. Society 1 (1952), 187-246 (Russian).

I. Parassidis: TEI of Larissa, 41110 Larissa, Greece

E-mail address: parasidis@hol.gr

P. Tsekrekos: Department of Mathematics, National Technical University of Athens, Zografou Campus, 15780 Athens, Greece

E-mail address: ptsekre@math.ntua.gr 


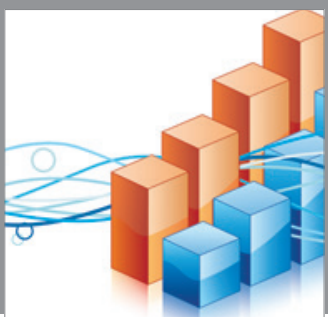

Advances in

Operations Research

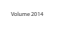

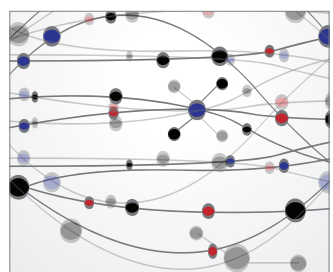

\section{The Scientific} World Journal
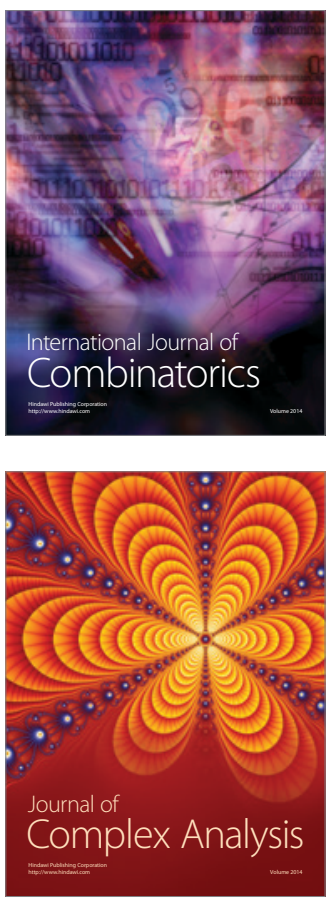

International Journal of

Mathematics and

Mathematical

Sciences
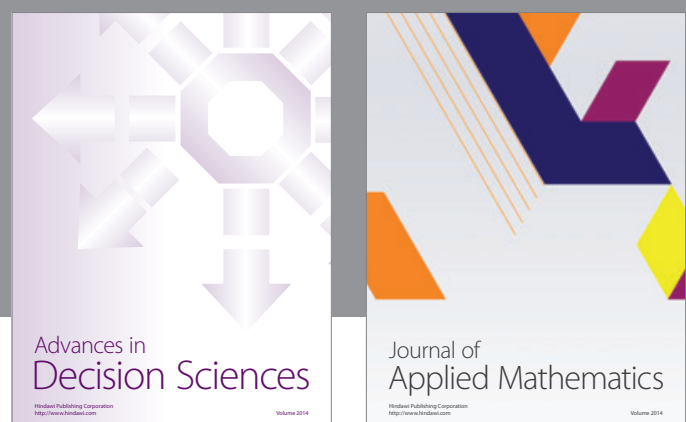

Journal of

Applied Mathematics
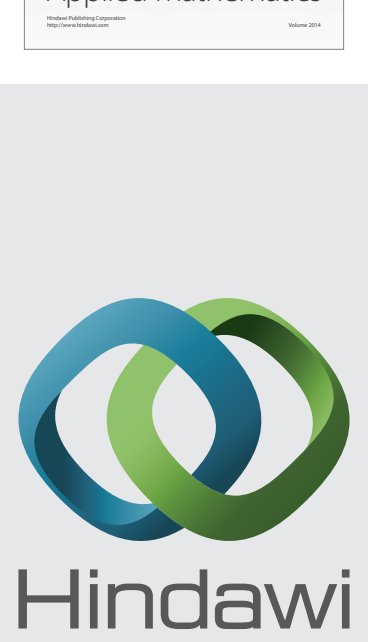

Submit your manuscripts at http://www.hindawi.com
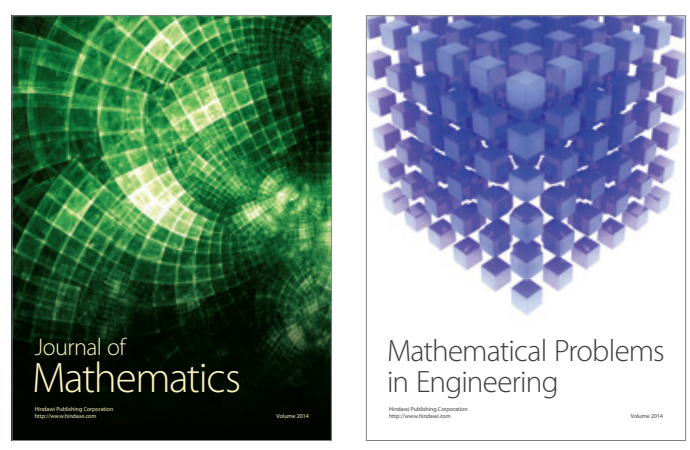

Mathematical Problems in Engineering
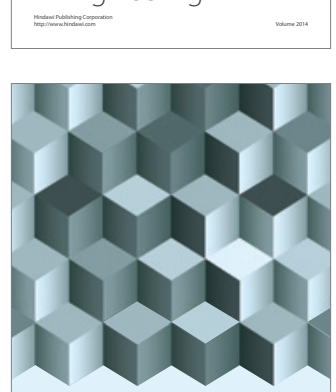

Journal of

Function Spaces
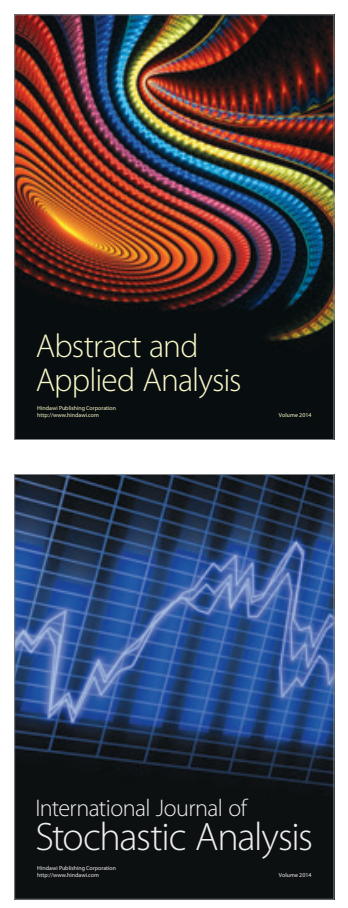

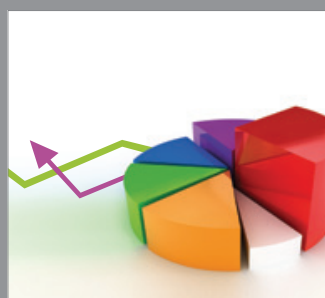

ournal of

Probability and Statistics

Promensencen
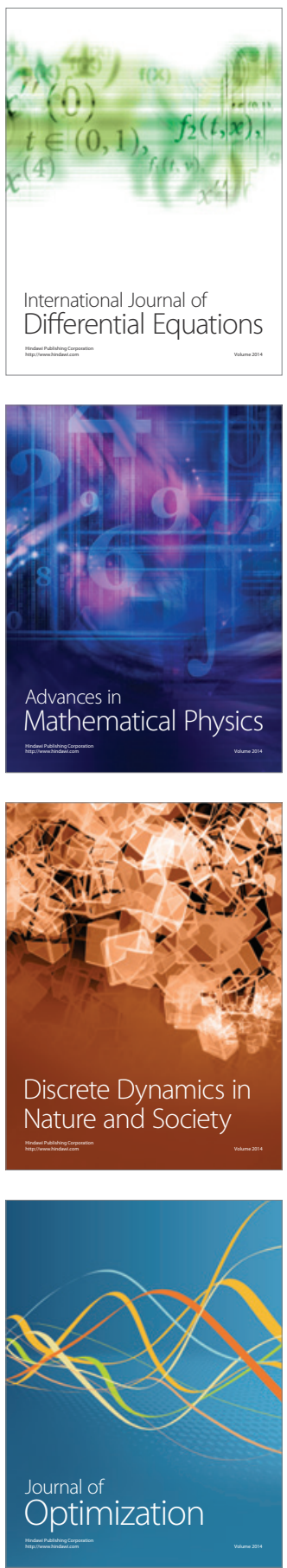